\title{
Propagation and Entrapment of Hydrocarbons in Porous Media under Capillarity Controlled Phase Alteration Conditions: A Visual Nanofluidics Analysis
}

\author{
Ilyas Al-Kindi and Tayfun Babadagli ${ }^{1}$ \\ University of Alberta \\ 7-203 Donadeo Innovation Centre for Engineering \\ 9211 - 116 Street NW \\ Edmonton, Alberta, Canada T6G $1 \mathrm{H} 9$
}

\section{Supporting Information}

Six video clips of the selected experiments:

Clip 1: The footage shows the initial vaporization of pure pentane in a homogeneous microfluidic chip, featured with uniform pore throat sizes (Fig. 11). Owing to the constant capillary pressure, the gas displacement took place consistently throughout the porous medium and the vapour propagation was quicker than what was observed with the heterogeneous microfluidic model, as shown in Fig. 11 in the manuscript and Clip 1a. Due to the confinement effect, the boiling temperature was $1.6 \%$ lower the normal boiling point $\left(35^{\circ} \mathrm{C}\right)$.

Clip 1a: The clip shows a captured footage of the initial vaporization of pure pentane in a heterogeneous micromodel (Fig. 11). The heterogeneity of capillary pressure resulted in a gas fingering and non-uniformity of microscopic vapour displacement. Wide deviations of capillary pressure in the system led the vapour movement and displacement to become slower than what was observed in the homogeneous models. Due to the confinement effect, the boiling temperature was $2.3 \%$ lower the normal boiling point $\left(35^{\circ} \mathrm{C}\right)$.

Clip 2: The footage shows the initial vaporization of pentane-heptane mixture in a homogeneous microfluidic model (Fig. 13). Despite the homogeneity of capillary pressure, a non-uniform microscopic gas displacement was observed due to the presence of a liquid phase which restricted the vapour flow significantly. Moreover, the viscous forces of the liquid phase acted as a barrier against the vapour flow which was the main cause of gas fingering as shown in Fig. 8 in the manuscript. Due to the confinement effect, the bubble point temperature was $6.7 \%$ lower than the bulk value $\left(54^{\circ} \mathrm{C}\right)$.

\footnotetext{
${ }^{1}$ Corresponding author, e-mail: tayfun@ualberta.ca
} 
Clip 2a: The footage presents the vaporization of pentane-heptane mixture in a heterogeneous micromodel (Fig. 13). The non-uniformity of capillary pressure and presence of a liquid phase in the porous medium drastically restricted the vapour flow within the pore throats thus making it considerably slower, resulting in gas channeling and less gas displacement. Due to the confinement effect, the bubble point temperature was $6.9 \%$ lower the bulk value $\left(54^{\circ} \mathrm{C}\right)$.

Clip 3: This clip shows the vaporization of the pentane-heptane-octane mixture in a homogeneous model (Fig. 14). As a result of the presence of the liquid phase with higher density, the gas propagation in the porous medium was less than the case with pure pentane, and an excessive gas fingering was observed due to the viscous forces in the liquid phase. The bubble point temperature was $3.9 \%$ less than the bulk value $\left(65^{\circ} \mathrm{C}\right)$ due to the confinement effect.

Clip 3a: The footage shows the vaporization of pentane-heptane-octane mixture in a heterogeneous model (Fig. 14). Owning to the heterogeneity of capillary pressure and presence of a liquid phase, a slow gas displacement and excessive gas channeling were noticed in the model. The bubble point temperature was $4.8 \%$ less than the bulk value $\left(65^{\circ} \mathrm{C}\right)$ due to the confinement effect. 